Témoigner Témoigner. Entre histoire et mémoire

Getuigen Revue pluridisciplinaire de la Fondation Auschwitz

$122 \mid 2016$

Révisionisme et négationisme

\title{
Négationnisme et concurrence des victimes
}

Negationism and competitive victimhoods

Negationisme en slachtofferconcurrentie

Valentina Pisanty

Traducteur : Benedicte Cavanna

\section{(2) OpenEdition}

Journals

Édition électronique

URL : https://journals.openedition.org/temoigner/4380

DOI : 10.4000/temoigner.4380

ISSN : 2506-6390

Éditeur :

Éditions du Centre d'études et de documentation Mémoire d'Auschwitz, Éditions Kimé

Édition imprimée

Date de publication : 2 mai 2016

Pagination : 101-112

ISSN : 2031-4183

Référence électronique

Valentina Pisanty, « Négationnisme et concurrence des victimes », Témoigner. Entre histoire et mémoire [En ligne], 122 | 2016, mis en ligne le 30 septembre 2021, consulté le 04 janvier 2022. URL : http:// journals.openedition.org/temoigner/4380 ; DOI : https://doi.org/10.4000/temoigner.4380

Tous droits réservés 


\section{Négationnisme et concurrence des victimes}

\author{
$\rightarrow$ Valentina Pisanty \\ Université de Bergame
}

(1) Pierre Vidal-Naquet, Les Assassins de la mémoire, Paris, La Découverte, 1987.

(2) Pour un examen rigoureux des stratégies rhétoriques des négationnistes, je suggère de consulter : Vidal-Naquet, ibid. ; Nadine Fresco, « Les Redresseurs de Morts ", in Les Temps Modernes, n 407, 1980 ; JeanClaude Pressac, Les Crématoires d'Auschwitz. La machinerie du meurtre de masse, Paris, CNRS, 1993 ; ainsi que mon ouvrage : Valentina Pisanty, L'irritante questione delle camere a gas: logica del negazionismo, Milano, Bompiani, 1998.

(3) McLuhan, interview au Corriere della sera du 23 mars 1978. enégationnisme est un dispositif rhétorique doté d'une logique interne, d'un répertoire de motifs récurrents et d'un arsenal de techniques argumentatives ayant pour but de semer le doute sur la réalité de l'extermination nazie.

On peut l'analyser en tant qu'objet sémiotique particulier en démontant les stratégies interprétatives et discursives dont se servent les «Eichmann de papier» - l'épithète est de Pierre Vidal-Naquet ${ }^{1}$ - pour soutenir que la Shoah est une invention de la propagande sioniste ${ }^{2}$. Mais un dispositif rhétorique n'agit pas dans le vide. Il n'existerait pas, si ce n'est dans la tête et dans les discours d'un nombre restreint de personnes, sans un auditoire réceptif, sans un système culturel disposéà l'accueillir, ne fût-ce que pour le blâmer ou le censurer. D’où il s'ensuit que le négationnisme a besoin de quelqu'un qui en assure le fonctionnement, en amorçant les circuits de communication qui le rendent efficace. Si, à titre d'hypothèse, on le désamorçait, comme avait suggéré de le faire McLuhan à propos du terrorisme des années de plomb en Italie ${ }^{3}$, il disparaîtrait en tant que phénomène social, ou du moins il serait à nouveau relégué au sein des circuits semi-privés de ses partisans actifs.

Dans cet article, je porterai mon attention sur certaines dynamiques culturelles initiées par le négationnisme dès sa première apparition sur la scène médiatique au cours de l'hiver 1978-1979, lorsqu'explosa en France le cas Faurisson. Pourquoi les négationnistes se sont-ils imposés à l'attention publique précisément à cette occasion, après trente ans d'activités souterraines ? Comment ont-ils été présentés par les médias, quel rôle ont-ils conquis sur la scène publique et comment sont-ils parvenus à élargir leur auditoire compte tenu du fait que le négationnisme avait été jusqu'alors un phénomène marginal sur le plan culturel, capable tout au plus de passionner quelques nostalgiques du nazifascisme?

L'hypothèse est la suivante : les négationnistes ont commencé à conquérir un auditoire plus large lorsqu'ils ont appris à exploiter à leur propre avantage les polémiques sur la mémoire qui faisaient rage à l'époque en Europe et aux États-Unis. Il s'agissait de conflits concernant, d'une part, le caractère présumé unique et incommensurable de la Shoah et, d'autre part, l'apparition de mémoires concurrentielles qui contestaient cette primauté - à l'aide de formats narratifs calqués sur le modèle qui avait permis à l'histoire du génocide juif de s'affirmer au sein de l'Encyclopédie 
Négationnisme et concurrence des victimes (suite)

collective. Ce climat d'intense revendication a facilité la pénétration de la thèse négationniste dans les médias : elle se faufilait dans les discours sur la mémoire, comme une roue faisant partie d'un engrenage plus complexe, dont la cohésion serait assurée par une conception patrimoniale de la mémoire, considérée comme une ressource à valoriser en fonction des intérêts de ses gestionnaires.

\section{LE BIG BANG DU CAS FAURISSON}

Il existe une coïncidence significative des dates. Les premières lettres de Faurisson dans Le Matin ( $1^{\mathrm{er}}$ novembre 1978) et dans Le Monde (16 décembre 1978 et 29 décembre 1978) furent publiées précisément au cours des mois où la presse occidentale s'échauffait sur les mérites ou les démérites de la mini-série télévisée Holocaust, l'événement médiatique qui - selon beaucoup - a contribué plus que tout autre à former la perception collective de la Shoah ${ }^{4}$, en projetant celle-ci au centre de l'Encyclopédie et des consciences, après des décennies caractérisées par un refoulement relatif, ou une « décantation», du traumatisme. Le lancement de cette adaptation télévisée - transmise par la NBC (n.d.t. groupe audiovisuel américain National Broadcasting Company) du 16 au 19 avril 1978 - fut accompagné de débats animés, qui concernaient, d'une part, les contenus historiques du téléfilm (le sort des Juifs d'Europe et les origines du mal nazi) et se concentraient, d'autre part, sur la qualité de l'expression télévisée de ces questions et sur la légitimité même d’une opération outrageusement commerciale qui réduisaitl'histoire de l'extermination à des formats narratifs éprouvés de façon à la rendre plus accessible à un public de masse. Les réactions critiques se polarisèrent aussitôt. Les uns voyaient dans cette mini-série une appropriation effrontée par le show-business américain d'une expérience traumatique que seuls ceux qui avaient «été là » avaient le droit de raconter. Les autres, au contraire, considéraient que la mise en spectacle - avec tous les risques de banalisation que cela comportait - était le prix à payer pour que la mémoire du génocide sorte des limites étroites de la communauté des victimes

(4) « More information about the Holocaust was imparted to more Americans over those four nights than over all the preceding thirty years " ("Ces quatre soirées ont fourni à bien des Américains beaucoup plus d'informations sur la Shoah que toutes celles des dernières trente années "), affirme Peter Novick in The Holocaust in American Life, Boston, Houghton Mifflin, 1999, p. 209.

(5) Claude Sarraute, «Les simplifications nécessaires », Le Monde du 2 mai 1978. et devienne un patrimoine de l'humanité tout entière. Voici comment, en France, Claude Sarraute résumait les termes de cette polémique destinée à durer des mois et à nourrir le vieux débat sur l'impossibilité de représenter la Shoah :

Où commence, où s'arrête le sens du sacré? Peut-on tout dire, tout simplifier, tout épeler à l'intention des masses, y compris l'indicible ? Faut-il courir, par souci d'efficacité, le risque de vulgariser, de banaliser le mal absolu, l'horreur totale ? Est-ce insulter, est-ce honorer les martyrs de l'holocauste que d'évoquer leur sort en images d'Épinal ? A-t-on le droit, en pareil cas, de mêler la fiction à la réalité ${ }^{5}$ ?

Selon les amateurs de cette historicalfiction, et notamment Sarraute elle-même, la leçon d'histoire donnée par Holocaust était vraiment nécessaire au public amé- 
ricain, qui avait des connaissances tellement vagues au sujet de l'extermination qu’il lui était arrivé de la minimiser ou de la nier. C'était là, d’après la journaliste, une préoccupation qui troublait plusieurs Juifs américains. Parmi eux, le rabbin Marc Tannenbaum (directeur du département des affaires interreligieuses auprès de l'Anti Defamation League, et également expert-conseil de la NBC pour la production de ce téléfilm) lui avait appris qu'aux États-Unis, un grand nombre de personnes considérait la Shoah comme une idée fixe de la communauté juive, comme s'il s'agissait d'une demande fastidieuse d'attention ou, pire encore, d'un chantage visant à promouvoir la cause sioniste. Pour confirmer ses dires, Tannenbaum relatait les activités de certaines organisations néo-nazies américaines (dirigées par la Christian Defense League) qui, durant la diffusion de Holocaust, avaient placé devant les studios de la NBC à Bâton-Rouge, en Louisiane, des piquets munis de pancartes niant l'extermination. Ils avaient aussi bombardé la chaîne de centaines d'appels téléphoniques de protestation ${ }^{6}$.

En soi, les manifestations néo-nazies auraient été peu de chose, vu le nombre restreint de participants. Et pourtant, plus elles donnaient corps à la peur d'une haine antisémite encore bien vivante, plus elles paraissaient scandaleuses (donc dignes d'entrer dans la chronique). Attirés par l'audience de la NBC, les militants de la Christian Defense League s'étaient amassés là où la visibilité leur était garantie par l'imposante machine publicitaire organisée pour le lancement de la mini-série. Ils étaient ravis d'incarner le rôle d'héritiers de Dorf et des autres nazis télévisés, tout en refusant d'être qualifiés d'antisémites. L'accumulation des intérêts et des diatribes qui éclateront dans le Big Bang du cas Faurisson quelques mois plus tard apparait ici en germe. D’une part, on trouve producteurs et éducateurs, qui convergent dans la mise au point d'une version passionnante de l'histoire génocidaire, riche en avertissements et en leçons à valeurs universelles, facilement assimilables. D’autre part, il y a les remontrances des critiques et de témoins influents, opposés à l'exploitation commerciale et rhétorique d'un traumatisme encore vivant et douloureux dans la mémoire des victimes. Il y a enfin les propos des racistes, aux prises avec la mémoire d'un crime historique indiscutablement affreux, dont le refoulement était indispensable pour relancer la vieille thèse du complot juif. Et s'il est vrai que la dissémination des connaissances historiques pouvait passer comme remède contre l'ignorance diffuse sur laquelle le racisme et l'antisémitisme tendent à prendre racine, il est tout aussi vrai que l'aspect spectaculaire, dans lequel ces connaissances furent enveloppées, excitait l'exhibitionnisme des négateurs et la curiosité de ceux qui avaient évité jusqu'alors de leur prêter trop d’attention.

Mais ce ne fut pas lors de cette circonstance particulière que les négationnistes s'imposèrent à l'attention internationale : quelques jours plus tard, l'incident était classé par les médias américains, habitués à cohabiter avec les effets collatéraux du principe de la liberté d'expression ratifié dans leur Constitution. Ce fut la rédaction de L'Express, dirigée à l'époque par Jean-François Revel, qui mit le feu aux
(6) Pour un compte-rendu de ces manifestations, voir l'édition du New York Times du 17 avril 1978, "Pickets at TV Studio Protest "Holocaust" ". 
Négationnisme et concurrence des victimes (suite)

(7) « Le mensonge », Le Monde du 31 octobre 1978.

(8) « Une lettre de MM. Raymond Aron et JeanFrançois Revel », Le Monde du $1^{\text {er }}$ novembre 1978.

(9) Elie Wiesel, «Trivializing the Holocaust ", New York Times du 16 avril 1978. poudres six mois plus tard (28 octobre -4 novembre 1978), en publiant l'interview par Philippe Ganier-Raymond de Louis Darquier de Pellepoix, ancien Commissaire général aux questions juives sous Vichy, condamné à mort par contumace en 1947 et résidant depuis lors officiellement en Andalousie. «ÀA Auschwitz seules les puces ont été gazées », déclarait le responsable de la rafle du Vélodrome d'Hiver. Comme on pouvait s'y attendre, le scandale éclata. Pourquoi donner la parole à un criminel impuni et impénitent, qui soutenait de façon provocatrice la thèse du complot juif et niait l'existence des chambres à gaz, alors qu'aucun événement dans l'actualité n'était susceptible de solliciter cette exhumation (à part le lien indirect avec les accords de Camp David du 17 septembre 1978) ? Et pourquoi juste à ce moment-là? Simone Veil, ministre de la Santé et de la Famille à l'époque, stigmatisa le scoop de L'Express, en l'inscrivant dans une tendance plus générale à relativiser les crimes nazis et à minimiser les fautes de Vichy. Sa position fut suivie par Pierre VianssonPonté $^{7}$ et par le Conseil des Communautés juives de France, dans un communiqué du $1^{\mathrm{er}}$ novembre 1978 qui suggérait que les déclarations de Darquier étaient à inscrire «dans le cadre d'une campagne inquiétante et orchestrée dans le but de banaliser le racisme et de minimiser l'horreur du génocide»; ces accusations furent vigoureusement rejetées par Revel et par Raymond Aron, son collaborateur à $L$ 'Express, qui précisèrent que l'intention de l'interview était au contraire de «mettre en évidence cette pathologie de la pensée humaine qu'est le racisme, comme on met en évidence un tissu cancéreux ${ }^{8}$.» Le jour même, Le Matin publia la première lettre de Faurisson.

Entretemps, contrairement à la troisième chaîne de la République fédérale allemande, les trois chaînes de la télévision française hésitaient à acquérir les droits de la mini-série de NBC. Cela fit également l'objet de véhémentes discussions, qui se référaient souvent au cas Darquier, pour argumenter les positions favorables ou contraires à la programmation du feuilleton historique en France. Simone Veil en recommanda la diffusion comme antidote à la maladie de la relativisation, dont l'interview de L'Express était selon elle le symptôme le plus évident. Le Premier ministre Raymond Barre, au contraire, mit en garde les directeurs de chaînes contre les pièges de la spectacularisation et les exhorta à traiter ces thèmes si délicats avec la plus grande vigilance : cette prudence fut interprétée par certains comme une réticence à promouvoir un débat public sur un des chapitres les plus honteux de l'histoire française récente, enseveli sous les célébrations d'auto-absolution de la Résistance, tandis que d'autres l'attribuèrent à un antiaméricanisme irréfléchi ou peut-être à un sentiment voilé d'antisionisme. Était-ce juste de diffuser le téléfilm américain ? Était-ce juste de publier l'interview de Darquier? À laquelle des deux formes de banalisation (celle qui spectacularise ou celle qui relativise) était-il indispensable de ne jamais céder? Ou aurait-il mieux valu cadenasser ces questions et laisser la parole uniquement aux survivants, comme l'avait déjà suggéré Elie Wiesel dans sa célèbre et acerbe critique parue dans le New York Times du 16 avril $1978^{9}$ ? Les réserves tombèrent le 30 novembre 1978, quand Antenne 2 annonça son intention d'acquérir les droits d'Holocaust. Comme pour démontrer l'urgence morale de cette 
décision (mais de fait en contribuant à la créer), Le Monde publia deux semaines plus tard la deuxième lettre de Faurisson.

NIER, BANALISER, SACRALISER

Il faut survoler la chronique des événements pour pouvoir y discerner un éventuel schéma, un engrenage rhétorique, une dynamique récurrente. Le négationnisme s'insinue dans les méandres d'un débat plus général sur les us et les abus de la mémoire ${ }^{10}$. C'est précisément au moment où celle-ci devient l'objet d'énormes investissements économiques, passionnels et politiques, renforcés par les intérêts conflictuels du présent, que se mettent en route des mécanismes de concurrence entre des sujets qui veulent en assumer le contrôle pour les motifs les plus divers. Chacun accuse l'autre - peu importe si cette accusation est justifiée, même si elle est sincère - de banaliser la mémoire, autrement dit de la soumettre à ses propres objectifs spécifiques, qu'ils soient de nature commerciale ou idéologique. Et comme les accusations de banalisation contiennent souvent une interdiction de sacralisation, la mémoire disputée se voit entourée d'une barrière défensive, sous forme d'interdits et de prescriptions, qui en contrôlent les modalités d'accès. Ainsi prend forme une catégorie de personnes autorisées à passer au crible les discours d'autrui pour décider lesquels sont légitimes et lesquels ne le sont pas, lesquels peuvent être jugés appropriés et lesquels doivent au contraire être rejetés comme recours illicites à l'histoire. Des associations d'anciens déportés, d'éminents personnages, des institutions culturelles, des organismes gouvernementaux se disputent ce rôle de gardien de la mémoire. Souvent en désaccord entre eux, ils sont mus par la volonté de protéger la mémoire des incursions de ceux qui, pour divers motifs, ont intérêt à la saccager, à l'exploiter, à la profaner voire à l'effacer. Les négationnistes se faufilent dans ces diatribes pour se frayer un chemin dans les médias. Les chassés-croisés de condamnations et de vetos préparent le terrain aux déclarations par lesquelles Faurisson conquiert le rôle de provocateur absolu. Ses interventions lui permettent de centraliser l'indignation, les peurs et les ressentiments soulevés par les polémiques des jours précédents. Classées dans la catégorie désormais familière de «controverses sur la Shoah", les thèses négationnistes trouvent un espace dans les palimpsestes journalistiques, toujours au nom du principe discutable qui veut que le traitement des pathologies passe par leur exhibition. Mais l'étalage du phénomène (ainsi que des inévitables protestations qu'il soulève), loin de le vaincre, ne fait que l'accroître. Le négationnisme est en quelque sorte légitimé par les tentatives de l'exorciser à coups d'anathèmes et de censures. Plus on l'attaque et plus il se renforce d'un point de vue rhétorique, il s'alimente de l'indignation qu'il provoque. Même les dénonciations les plus vibrantes, comme celles des trente-quatre historiens français publiées dans Le Monde du 21 février 1979, obtiennent l'effet non souhaité de déplacer l'accent de la polémique sur le problème de la liberté de parole. «Il n’y a pas, il ne peut pas y avoir de débat sur l'existence des chambres à gaz » : cette malencontreuse formulation confond un refus historiographique plus que légitime d’admettre les négationnistes dans la
(10) Je me suis déjà occupée des rapports polémiquement solidaires entre la négation, la banalisation et la sacralisation dans l'essai suivant : Valentina Pisanty, Abusi di memoria, Milano, Bruno Mondadori, 2012. 
Négationnisme et concurrence des victimes (suite)
(11) Sur la négation du génocide arménien, voir Richard G. Hovannisian, Remembrance and Denial: The Case of the Armenian Genocide, Detroit, Wayne State University Press, 1999. sphère des interlocuteurs possibles (inutile de discuter avec les charlatans) avec un interdit moral discutable (que personne ne se hasarde à douter de l'extermination) qui provoque à son tour de nouvelles controverses et d'autres cycles de revendications. De nouvelles voix se font entendre, éveillées par ce dilemme scabreux : faut-il bâillonner les crapules ? Est-ce plus intolérable que quelqu'un nie les chambres à gaz ou que quelqu'un d'autre l'empêche de le faire ? Et pourquoi d'autres négations historiques ne suscitent-elles pas de telles levées de boucliers? Ou ne sommes-nous pas confrontés ici à la énième expression de ce «particularisme juif» dont plusieurs parlent tout bas, mais dont peu osent parler ouvertement?

\section{COMPARAISONS}

Le négationnisme de la Shoah n'est pas seul à exister. D’autres crimes historiques, comme le génocide arménien (1915-1916), les massacres de Nankin (1937) et celui de Katyń (1940), ont été enterrés dans l'immédiat, puis systématiquement et durablement niés. Le cas le plus débattu, du moins en Occident, est la négation du génocide arménien auquel l'identité nationale turque est tenacement accrochée. Depuis la fin des années 1960, des auteurs comme Kamuran Gürün, Stanford Shaw, Justin McCarthy et Bernard Lewis ont soutenu le gouvernement d'Ankara par des articles et des essais qui valorisaient la thèse officielle selon laquelle l'évacuation de centaines de milliers d'Arméniens du haut plateau d'Anatolie ne serait pas dérivée d’un plan précis d'anéantissement voulu par le régime des Jeunes Turcs, mais plutôt d'une réimplantation des Arméniens rendue nécessaire par leur terrorisme insurrectionnel. Il est vrai que certains réfugiés seraient morts de maladies et de privations durant le trajet vers la Syrie et la Mésopotamie, mais la plupart d'entre eux auraient survécu au déplacement pour reconstruire ailleurs une existence prospère.

Les affinités entre ces arguments et cette méthode avec ceux visant à nier la Shoah sautent aux yeux ${ }^{11}$. Ils incluent la réduction drastique du nombre des victimes, le désaveu du projet d'extermination, la délégitimation des témoignages et des documents à charge, ainsi que le recours à des thèses basées sur des complots pour expliquer la persistance du «mythe » génocidaire. Les principales différences concernent en revanche la structure et les dynamiques du pouvoir qui est à la tête du dispositif négationniste. Quand c'est l'État qui nie, et non pas des groupes de fanatiques isolés, alors la mémoire est amputée au moyen de la censure, du refoulement forcé et de la répétition insistante de vérités présentées comme étant sans appel. Les rôles de celui qui nie et de celui qui sacralise (l'autorité qui établit ce qu'il faut et ce qu'il ne faut pas dire) coïncident, et même la banalisation (qui tend ici à réduire et à relativiser) sert à soutenir la narration voulue par l'hégémonie. Les trois roues de cet engrenage sont manœuvrées par une seule volonté, contrairement à ce qui se passe avec le négationnisme de la Shoah, opposé de façon polémique (tout en étant solidaire du point de vue du système) aux autres dispositifs rhétoriques qui se disputent la gestion de la mémoire. 
Pour s'opposer au négationnisme d'État, il ne suffit pas de fournir les preuves documentaires de l'événement nié, comme on le ferait devant un tribunal universel. En l'absence d'un auditoire réceptif, toute reconstruction historique tombe dans le vide. C'est pourquoi celui qui veut briser le mur du silence a besoin d'un contexte politique et culturel disposé à recueillir ses thèses. Mais il doit également réussir à comprimer sa contre-mémoire dans des formats de communication capables de passionner, d'être user-friendly et efficaces dans ce contexte déterminé. Une forme de banalisation qui soit en fait spectaculaire, mais qui est indispensable pour pouvoir transformer le souvenir confus des événements refoulés en un récit fort et tendu, capable de tenir tête à l'historiographie du régime.

Et c'est ce qui se passe en 1965, le 24 avril, à l'occasion du cinquantième anniversaire des premières arrestations opérées au sein de l'élite arménienne de Constantinople. À Erevan, capitale de la République démocratique d'Arménie (qui faisait partie à l'époque de l'Union soviétique), des milliers de manifestants se réunissent pour demander au Kremlin de reconnaître le génocide perpétré. Les manifestations se propagent dans le monde entier, y compris aux États-Unis, où cet éveil d’attention donne naissance à de nombreuses initiatives de différentes natures. Parmi celles-ci, citons la publication dans le New York Times d'un éditorial qui, d'une part, tend la main à la communauté arménienne (tout en l'exhortant au pardon) et, d'autre part, lui déconseille de comparer les deux massacres :

Armenians do in fact compare the event to the Hitlerite genocide of the Jews. The numbers massacred by the Turks will never be known. Armenian estimates running as high as 2,000,000. The English historian H. A. L. Fisher sets the figure at 1,000,000. It is terrible enough, although the comparison with Hitler's genocide is not quite accurate. Hitler exterminated Jews simply on racist grounds ; in the Turkish extermination of the Armenians, whom they considered a threat to the Ottoman Empire, there was indeed an element of racism, but the primary factor was nationalism. And many Turks also lost their lives in the holocaust $\mathrm{t}^{12}$.

Que peut-on déduire de cette brève reconstruction ? D’abord, pour conquérir un auditoire auprès des médias internationaux, la communauté arménienne d'Amérique traduit son traumatisme dans le lexique du génocide juif. L'histoire de la Shoah (qui en 1965, suite au procès Eichmann, commence à prendre pied dans les consciences) offre un appui, un modèle, un schéma intelligible qui permet d'interpréter les faits de 1915 de façon rétrospective. L'assimilation à ce modèle plus accessible provoque une forte impression. C'est sous cette forme que le génocide arménien commence à se frayer un chemin dans l'Encyclopédie, en attribuant de fait à ceux qui commirent le massacre le rôle (du reste reconnu par Hitler luimême) de précurseurs des nazis. C'est si vrai que depuis les années 1970, face à l'insuffisance des instruments traditionnels de la censure, le gouvernement turc va intensifier ses efforts, en engageant les académiciens alignés sur le pouvoir dans
(12) «Armenia Remembers », New York Times, 24 avril 1965.

Traduction en fin d'article. 
Négationnisme et concurrence des victimes (suite)

(13) Jean-Michel Chaumont, La concurrence des victimes, Paris, La Découverte, 2002. une campagne négationniste appelée à devenir, au cours des décennies suivantes, toujours plus pesante et plus technique. L'autre aspect saillant est la réaction pointilleuse du New York Times, qui tient à préciser les différences entre les deux exterminations pour éviter toute possibilité d'assimilation. Le ton est poli, mais le message est clair : ne banalisons pas, s’il vous plaît, le crime d'Hitler est unique et incomparable.

Dans le discours journalistique, les subtilités ont tendance à se perdre en chemin et, de même que la comparaison se transforme souvent en égalisation, l'opération inverse - celle de souligner les différences - peut, elle aussi, assumer les tons d'un jugement de valeur : si x et y sont différents, cela signifie que x est plus z que l'autre. On voit apparaître une sorte de palmarès des massacres, comme si un massacre opéré sur base nationaliste était moins grave - plus justifiable ou du moins plus ordinaire - qu'une extermination effectuée pour des motifs purement racistes. On pourrait discuter longuement de cette thèse, sans beaucoup de sens par ailleurs, mais qui, aux yeux de ceux qui ont subi ces violences, pourrait laisser entendre qu'il existe un classement insensé des souffrances : notre génocide est pire que le vôtre, les maux qui vous ont été infligés - même les plus terribles - n’appartiennent pas au même ordre que les nôtres.

\section{LA CONCURRENCE DES VICTIMES}

L’on entrevoit le germe du phénomène que Jean-Michel Chaumont désignera sous le nom de « concurrence des victimes ${ }^{13}$ » - une compétition perverse entre les mémoires dolentes et revendicatives, qui se disputent la «palme de la souffrance »et pour laquelle la Shoah, au fur et à mesure qu'elle s'élève au rôle d'événement charnière de l'histoire du XX $\mathrm{XX}^{\mathrm{e}}$ siècle, représente le terme de comparaison désormais évoqué de façon constante. Ceux qui contestent la thèse de la singularité de la Shoah sont souvent poussés par l'intention polémique de dénier aux Juifs le statut «privilégié» de victimes absolues et réclament pour leur propre groupe de référence un titre honorifique analogue. Et vice versa, les défenseurs les plus infatigables de la thèse des victimes absolues revendiquent la primauté de la souffrance subie par les Juifs sous le nazisme et accusent de banalisation sacrilège toute comparaison avec d'autres persécutions, ce qui ne fait que raviver les attaques d'exclusivisme victimaire dont ils font l'objet.

Il en découle une cascade de polémiques ultérieures qui font boule de neige dans les années 1980, dans la foulée du succès de Holocaust et toutes concernent la prétendue singularité du génocide juif.

To claim that the holocaust was unique can only imply that attempts to annihilate other national or cultural groups are not to be considered genocide, thus diminishing the gravity and moral implications of any genocide anywhere, any time. It also implies that the Jews 
have a monopoly on genocide, that no matter what misfortune befalls another people, it cannot be as serious or even in the same category as the Holocaust ${ }^{14}$.

Ainsi s'exprime Pierre Papazian dans un défoulement qui n’a certainement rien à voir avec le négationnisme, mais qui enfonce le couteau dans la plaie - lorsqu'il reproche aux Juifs, comme si ceux-ci formaient une catégorie compacte, de sacraliser leur propre génocide au détriment de ceux d'autrui. Pourquoi percevoir une telle offense dans le fait que le génocide juif soit considéré comme l'événement le plus traumatique de l'histoire de l'humanité dans l'absolu ? Parce que cette primauté porte à minimiser par contraste les tragédies d'autrui. Mais pourquoi, si la Shoah est considérée comme un événement « uniquement unique », étranger à toute série historique et par conséquent incommensurable, les autres massacres devraient-ils se mesurer avec elle et souffrir de cette confrontation? Voici le cœur du problème: le jugement d'incomparabilité coexiste avec le fait que la mémoire de la Shoah s'affirme de plus en plus comme un paradigme pour toutes les autres mémoires collectives. Dans le discours public, la Shoah est à la fois l'exception et la règle, un prototype paradoxal qui n'admet pas d'autres occurrences en dehors de soi.

Arrêtons-nous un moment sur cette aporie, qui provoque tant de controverses. Depuis que la Shoah a été élevée au rang d'événement exceptionnel dans l'histoire de l'humanité, tout autre événement traumatique a commencé à paraître (ou à être présenté) comme une dérivation, une approximation par défaut, un calque partiel de cet événement malheureusement exemplaire. De la Nakba à la Guerre au Liban, de l'ex-Yougoslavie aux attentats au World Trade Center, il n'est de conflit international de ces dernières décennies qui n'ait été filtré à travers le lexique de la Shoah, avec toutes les interprétations forcées et les effets de banalisation qu'un tel cliché comporte. Par ailleurs, les rapprochements plus ou moins banalisants provoquent des vagues d'indignation que les médias n'hésitent pas à amplifier. Le principe d'unicité est confirmé avec véhémence, tandis que sur la tête des banalisateurs plane l'accusation de profanation (antisémite) de la mémoire, et cela parfois sur la base d'arguments historiques, comme dans l'article du New York Times sur les Arméniens, d'autres fois par des prises de positions dogmatiques relatives à l'incomparabilité absolue d'un événement qui «transcende l'histoire». Tout aussi ponctuelles sont les réactions d'intolérance manifestées par ceux qui accusent les gardiens de la mémoire (identifiés à la hâte à la communauté juive internationale) de sacraliser la Shoah en fonction d'objectifs idéologiques propres et spécifiques (par exemple, celui d'exercer un monopole de la mémoire pour promouvoir la cause sioniste). L’escalade est inévitable.

Cette tempête ouvre aux négationnistes différentes possibilités de manœuvre. D’abord, ils concluent des alliances tactiques avec les banalisateurs, en misant sur l'aversion pour tout usage sacralisant de la mémoire. Pour comprendre comment, en partant de la dénonciation de la tendance juive à sacraliser la mémoire, on peut
(14) Pierre Papazian, « A “Unique Uniqueness"? », in Midstream, vol. XXX, n. 4, 1984, p. 14-18. Traduction en fin d'article. 
Négationnisme et concurrence des victimes (suite)

(15) Serge Thion, Vérité historique ou vérité politique, Paris, La Vieille Taupe, 1980.

(16) Ernst Nolte, Streitpunkte, Berlin, Propyläen, 1993. glisser vers l'aval idéologique du discours négationniste, présenté par Thion comme un défi courageux aux tabous historiographiques et à l'intolérable diktat de l'establishment académique, lisons cet extrait de Serge Thion et comparons-le à la citation de Pierre Papazian reportée ci-dessus :

Personne ne niera qu'il existe une sorte d'hésitation, ou même de censure, à l'égard de tout discours tenu sur les Juifs, ou sur des Juifs, ou sur le sionisme, ou sur Israël, si la parole émise n'a pas été d'abord, d'une façon ou d'une autre, autorisée. Pour l'écouter, il faut savoir, comme on dit, d'où on parle. Faute d'une sanction appropriée, d'une marque de légitimation, tout discours sur ce sujet est voué aux gémonies, livré au soupçon ${ }^{15}$.

Dans cette optique, l'iconoclastie des thèses négationnistes incarne déjà un bon motif de prêter attention à Faurisson (pour se demander "s'il existe une raison, fût-elle minime, de poser le problème des chambres à gaz en termes de faits historiques »), indépendamment de la validité de ses argumentations, sur lesquelles Thion préfère ne pas se prononcer.

Une forme de collaboration encore plus ambigüe sera celle favorisée par Ernst Nolte qui, dans sa tentative d'assimiler les crimes nazis à ceux des Soviétiques, consacre un chapitre interlocutoire de Streitpunkte ${ }^{16}$ à la «perspective du révisionnisme radical », c'est-à-dire du négationnisme, en soutenant que «le dernier mot n’a pas encore été dit dans les discussions [sur l'existence des chambres à gaz] entre les experts techniques. » Remarquons que Nolte n'arrive jamais à embrasser de façon explicite la thèse négationniste, qu'il semble au contraire refuser dans le premier chapitre de son livre : «Il ne peut y avoir de doute raisonnable quant à l'effectivité des mesures d'extermination à l'encontre du judaïsme allemand et européen.» Si ce n'est que, quelques pages plus loin, il rouvre la possibilité de douter, en légitimant des auteurs comme Stäglich, Roques, Leuchter et Mattogno, dont il loue la formulation scientifique, la bonne foi et la solidité des argumentations qu'il invite les lecteurs à prendre au sérieux, ne fût-ce qu'au nom du principe de la liberté d'expression. De telles ouvertures suggèrent qu'entre Nolte et les négationnistes s'instaure un jeu de rôle tacite, pas nécessairement conscient ; Nolte s’y limiterait à accréditer les négationnistes et à semer le doute sur la réalité de l'extermination, sans toutefois aller jusqu’à nier les chambres à gaz ( « seule exception » des crimes nazis par rapport aux crimes soviétiques) ; tandis que les négationnistes se chargent de la sale besogne de négation pure et simple qui, si elle devait passer, ôterait de l'Encyclopédie l'obstacle principal à l'égalisation complète entre les crimes nazis et les crimes soviétiques. Mais ce ne sont pas les banalisateurs, somme toute plutôt prudents et circonspects, qui poussent le plus les négationnistes vers le devant de la scène médiatique. Paradoxalement, ce sont plutôt les anathèmes les plus retentissants et les censures les plus sévères qui alimentent la machine de la négation. Depuis la déclaration contre Faurisson des trente-quatre historiens que nous avons abordée plus haut, jusqu’à la promulgation de lois spécifiques de la mémoire, les interdits sacralisants poussent les transgresseurs dans une zone clandestine de l'Encyclopédie, celle où une culture 
enferme ses monstres, mais ne les fait pas disparaitre : au contraire, elle les transforme en objets d'attention morbide. Et, de fait, chaque nouvelle application de la loi Gayssot, ou d'autres lois analogues, a pour effet de provoquer l'habituelle traînée de polémiques, remontrances, pétitions et lettres ouvertes par lesquelles le négationnisme marque sa présence dans les médias.

«Les punir ne servirait à rien d'autre qu’à en multiplier l'espèce », tel était l'avertissement de Pierre Vidal-Naquet en $1987^{17}$, qui avait prévu les sursauts de notoriété que l'application d'une loi anti-négationniste aurait offert à ses inculpés. Le fait de stigmatiser génère des identités contrastives ; et c'est pour cela que légiférer contre le négationnisme en tant que tel, et non au sujet des autres délits avec lesquels celui-ci se recoupe parfois, est le plus grand signal de reconnaissance que l'on puisse offrir à ceux qui cachent leur antisémitisme sous le manteau de principes démocratiques élevés.

Il est irresponsable de favoriser ces mécanismes. Ne pas parler avec les négationnistes, selon la recommandation de Vidal-Naquet, signifie aussi ne pas parler contre eux, ne pas les choisir comme adversaires directs. Au contraire, parler des négationnistes signifie les observer à bonne distance, voir comment ils se comportent, comprendre comment ils s'insèrent dans des engrenages historiques, politiques et culturels plus complexes. Cela équivaut à désacraliser le phénomène pour le priver de tout charisme diabolique et de tout attrait anti-establishment; cela signifie aussi d'en montrer la grossièreté, outre le sectarisme, et de s'activer en même temps afin que, dans l'Encyclopédie commune, les stratégies de déni se réduisent à des trucs bien connus par lesquels seul un lecteur exceptionnellement ingénu pourrait se laisser surprendre.

Traduction libre de la citation tirée du New York Times : «Les Arméniens comparent en fait leur génocide à celui des Juifs par Hitler. On ne connaîtra jamais le nombre des personnes massacrées par les Turcs, mais les estimations faites par les Arméniens arrivent au chiffre de 2 millions, tandis que l'historien anglais parle d'un million. C'est de toute façon terrible, mais la comparaison avec le génocide perpétré par Hitler n'est pas tout à fait juste. Hitler a exterminé les Juifs tout simplement pour des motifs raciaux; dans l'extermination des Arméniens par les Turcs, qui considéraient ceux-ci comme une menace pour l'Empire Ottoman, il y avait certainement une part de racisme mais le facteur prépondérant a été le nationalisme. Et bon nombre de Turcs ont perdu la vie lors de l'Holocauste.»

Traduction libre de la citation de Papazian : «Revendiquer l'unicité de l'Holocauste ne peut qu'impliquer le fait que toute tentative d'anéantissement d'autres groupes nationaux ou culturels ne doive pas être considérée comme un génocide, ce qui diminue la gravité et les implications morales de n'importe quel génocide, où et quand il est perpétré. Cela implique aussi que les Juifs aient un monopole sur le

(17) Op. cit. 
Négationnisme et concurrence des victimes (suite) génocide et qu'il importe peu quels malheurs affligent d'autres peuples, car aucun malheur ne peut être aussi sérieux que la Shoah ni même appartenir à la même catégorie que celle-ci. »

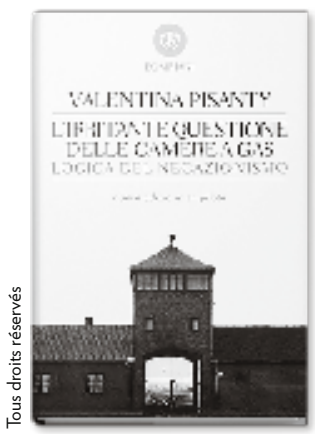

\section{Traduit de l'italien \\ par Benedicte Cavanna}

Cet article est une traduction partielle et retravaillée des pages 291-310 de la postface de la réédition de L'irritante questione delle camere a gas : logica del negazionismo (L'irritante question des chambres à gaz: logique du négationnisme).

\section{BIBLIOGRAPHIE}

- Benbassa, Esther (2007) : La souffrance comme identité, Paris, Fayard.

- Chaumont, Jean-Michel (2002) : La concurrence des victimes, Paris, La Découverte.

- Cohn Sherbok, Dan (dir.) (2002) : Holocaust Theology. A Reader, New York, New York University press.

- Flores, Marcello ; Levis Sullam, Simon ; Matard-Bonucci, Marie-Anne ; Traverso, Enzo (dir.) (2010) : Storia della Shoah in Italia. Vicende, memorie, rappresentazioni, Torino, UTET.

- Fresco, Nadine (1980) : «Les Redresseurs de Morts », in Les Temps Modernes, n 407.

- Giglioli, Daniele (2014) : Critica della vittima, Roma, Nottetempo.

- Halbwachs, Maurice (1950) : La mémoire collective, Paris, PUF.

- Hovanisian, Richard G (1999) : Remembrance and Denial: The Case of the Armenian Genocide, Detroit, Wayne State University Press.

- Nolte, Ernst (1993) : Streitpunkte, Berlin, Propyläen.

- Novick, Peter (1999) : in The Holocaust in American Life, Boston, Houghton Mifflin.

- Papazian, Pierre (1980) : «A Unique Uniqueness? », in Midstream, vol. XXX, n 4.

- Pisanty, Valentina (1998) : L'irritante questione delle camere a gas: logica del negazionismo, Milano, Bompiani, "Studi Bompiani, ll campo semiotico ».

- Pisanty, Valentina (2012) : Abusi di memoria, Milano, Bruno Mondadori.

- Poggio, Pier Paolo (1997) : Nazismo e revisionismo storico, Roma, manifestolibri.

- Pressac, Jean-Claude (1993) : Les crématoires d'Auschwitz. La machinerie du meurtre de masse, Paris, CNRS.

- Rusconi, Gian Enrico (dir.) (1987) : Un passato che non passa, Torino, Einaudi.

- Thion, Serge (1980) : Vérité historique ou vérité politique, Paris, La Vieille Taupe.

- Todorov, Tzvetan (1995) : Les abus de la mémoire, Paris, Arléa.

- Traverso, Enzo (2006) : Il passato. Istruzioni per l'uso, Verona, Ombre Corte.

- Türcke, Christoph (1987) : Gewalt und Tabu. Philosophische Grenzgänge, Lüneburg, Dietrich zu Klamplen Verlag.

- Vidal-Naquet, Pierre (1987) : Les Assassins de la mémoire, Paris, La Découverte. 